\title{
Dividend Payments with a Hybrid Strategy in the Compound Poisson Risk Model
}

\author{
Peng Li1, Chuancun Yin ${ }^{*}$, Ming Zhou ${ }^{2}$ \\ ${ }^{1}$ School of Mathematical Sciences, Qufu Normal University, Qufu, China \\ ${ }^{2}$ China Institute for Actuarial Science, Central University of Finance and Economics, Beijing, China \\ Email: lipengruby@gmail.com,
}

Received 15 April 2014; revised 25 May 2014; accepted 6 June 2014

Copyright (C) 2014 by authors and Scientific Research Publishing Inc.

This work is licensed under the Creative Commons Attribution International License (CC BY).

http://creativecommons.org/licenses/by/4.0/

(c) (i) Dpen Access

\begin{abstract}
In this paper, a hybrid dividend strategy in the compound Poisson risk model is considered. In the absence of dividends, the surplus of an insurance company is modelled by a compound Poisson process. Dividends are paid at a constant rate whenever the modified surplus is in a interval; the premium income no longer goes into the surplus but is paid out as dividends whenever the modified surplus exceeds the upper bound of the interval, otherwise no dividends are paid. Integrodifferential equations with boundary conditions satisfied by the expected total discounted dividends until ruin are derived; for example, closed-form solutions are given when claims are exponentially distributed. Accordingly, the moments and moment-generating functions of total discounted dividends until ruin are considered. Finally, the Gerber-Shiu function and Laplace transform of the ruin time are discussed.
\end{abstract}

\section{Keywords}

Hybrid Dividend Strategy, Compound Poisson Risk Model, Moment-Generating Function, Gerber-Shiu Function

\section{Introduction}

The dividends problem was first proposed by Finetti [1], who considered a discrete time risk model and found that the optimal dividend strategy is a barrier strategy, that is, any surplus above a certain level would be paid as dividend. Nowadays, this problem still attracts a lot of research interest. For example, [2] [3] considered the compound Poisson risk model. [4] studied the continuous counterpart of Finetti [1], and it is assumed that the surplus is a Brownian motion with a positive drift. Jeanblanc-Picque and Shiryaev [5] and Asmussen and Taksar

*Corresponding author. 
[6] postulated a modified version of barrier strategy called threshold strategy, that is, dividends are paid at a constant rate whenever the surplus is above a threshold level; however, when the surplus is below the threshold level, no dividends are paid. Some calculations for the classical risk model and Brownian motion model are given in [7] [8]. For recent publications on this topic, see, for example, [9]-[14].

Recently, the multi-layer dividend strategy as an extension of the threshold dividend strategy has drawn many authors' attention. Under such a dividend strategy, premiums will be collected at different rates whenever the surplus is in different layers. The modified surplus process is obtained from the original surplus process by refraction at each threshold level. Within this framework, many authors have studied the Gerber-Shiu expected discounted penalty function, see, for instance, [15]-[17] and the references therein.

Under such framework, $\mathrm{Ng}$ [18] combined barrier strategy and threshold strategy for the first time and then proposed a hybrid dividend strategy, who considered a dual risk model with phase-type gains under a hybrid dividend strategy and derived the explicit formula for the expected total discounted dividends until ruin and the Laplace transform of the time of ruin. In this paper, we consider the hybrid dividend strategy for the classical risk model. Let $b_{2}>b_{1}$ be two positive constants, under a hybrid strategy, no dividends are paid whenever the modified surplus is below the level $b_{1}$; dividends are paid at a constant rate $\alpha \quad(\alpha>0)$ whenever the modified surplus is in interval $\left(b_{1}, b_{2}\right)$; the premium income no longer goes into the surplus but is paid out as dividends whenever the modified surplus exceeds the level $b_{2}$. The modified surplus is obtained from the original surplus process by refraction at the level $b_{1}$ and reflection at the level $b_{2}$. The hybrid dividend strategy introduced above is a generalization of a pure barrier strategy and a pure threshold strategy. Apparently the hybrid strategy is more realistic than a pure barrier strategy, because it is inflexible for companies to use a switching mechanism of either paying nothing or paying all excess surplus as dividends. In the meantime, it is more practical than a pure threshold, because it is the ideal for a surplus of a company to be allowed to grow infinitely.

The rest of the paper is organized as follows. In Section 2, we find the integro-differential equations and boundary conditions for the expected discounted dividend payments until ruin. The integro-differential equations with boundary conditions satisfied by the moments and the moment-generating function are given in Section 3. Section 4 discussed the integro-differential equations with boundary conditions for the Gerber-Shiu function, and Section 5 presents the integro-differential equations with boundary conditions satisfied by the Laplace transform of ruin time.

\section{The Model}

We consider the compound Poisson model of risk theory with initial surplus $u>0$. In the absence of dividends, the surplus process $U_{t}$ at time $t$ is given by

$$
U_{t}=u+\mu t-S_{t} \equiv u+\mu t-\sum_{i=1}^{N(t)} Y_{i}, \quad t \geq 0,
$$

where $\mu$ is the premium rate, and $\left\{S_{t}\right\}$ representing the aggregate claims up to time $t, N(t)$ is a Poisson process with intensity $\lambda$, and $Y_{i}, i=1, \cdots$, independent of $\{N(t) ; t>0\}$, are positive i.i.d. random variables with distribution function $P(y)$ and density function $p(y)$.

Unlike the dividend strategies in [4] [8], we assume the company will pay dividends to its shareholders according to a hybrid dividend strategy with parameters $b_{2}>b_{1}>0$. The dividends consists of two parts. The first part of dividends are paid at a constant rate $\alpha \in(0, \mu)$ whenever the modified surplus between the level $b_{1}$ and the level $b_{2}$. The second part, whenever the modified surplus reaches the level $b_{2}$, the overflow will be paid as dividends. For $t>0$, let $D(t)=D_{1}(t)+D_{2}(t)$ denote the aggregate dividends paid by time $t$, where $D_{1}(t)$ and $D_{2}(t)$ caused by the two parts of dividends, respectively. Thus

$$
\tilde{U}_{t}=U_{t}-D(t) \text {, }
$$

is the company's modified surplus at time $t$.

Let $T$ be the ruin time of $\left\{\tilde{U}_{t} ; t \geq 0\right\}$, namely

$$
T=\inf \left\{t \geq 0 \mid \tilde{U}_{t}<0\right\},
$$

and let $\delta>0$ be the force of interest for valuation, we denote 


$$
D=\int_{0}^{T} \mathrm{e}^{-\delta t} \mathrm{~d} D(t), D_{1}=\int_{0}^{T} \mathrm{e}^{-\delta t} \mathrm{~d} D_{1}(t), D_{2}=\int_{0}^{T} \mathrm{e}^{-\delta t} \mathrm{~d} D_{2}(t) .
$$

We use the symbols $V\left(u ; b_{1}, b_{2}\right), V_{d}\left(u ; b_{1}, b_{2}\right), V_{r}\left(u ; b_{1}, b_{2}\right)$ to denote the expectations of $D, D_{1}, D_{2}$, i.e.

$$
V\left(u ; b_{1}, b_{2}\right)=E_{u}[D], V_{d}\left(u ; b_{1}, b_{2}\right)=E_{u}\left[D_{1}\right], V_{r}\left(u ; b_{1}, b_{2}\right)=E_{u}\left[D_{2}\right] .
$$

Define the moment-generating function of $D$ by

$$
M\left(u, z ; b_{1}, b_{2}\right)=E\left[\mathrm{e}^{\mathrm{zD}} \mid \tilde{U}_{0}=u\right] \equiv E_{u}\left[\mathrm{e}^{\mathrm{zD}}\right], u \geq 0,
$$

and $k$ th moment by

$$
V_{k}\left(u ; b_{1}, b_{2}\right)=E\left[D^{k} \mid \tilde{U}_{0}=u\right], u \geq 0, k \in N,
$$

with $V_{0}\left(u ; b_{1}, b_{2}\right)=1$, and the Gerber-Shiu functions by

$$
\Phi\left(u ; b_{1}, b_{2}\right)=E\left[\mathrm{e}^{-\delta T} \omega\left(\tilde{U}_{T-},\left|\tilde{U}_{T}\right|\right) I(T<\infty) \mid \tilde{U}_{0}=u\right], u \geq 0,
$$

where $\tilde{U}_{T-}$ is the surplus immediately before ruin, $\left|\tilde{U}_{T}\right|$ is the deficit at ruin and the penalty $\omega(x, y)$ is a nonnegative bounded measurable function of $x>0, y>0$, and the Laplace transform of ruin time by

$$
L\left(u ; b_{1}, b_{2}\right)=E\left[\mathrm{e}^{-\delta T} \mid \tilde{U}_{0}=u\right] \equiv E_{u}\left[\mathrm{e}^{-\delta T}\right], u \geq 0 .
$$

\section{Expected Discounted Dividend Payments}

In this section, we consider the hybrid dividend strategy for dividend payments in a compound Poisson risk model. We write

$$
\begin{aligned}
& V_{d}\left(u ; b_{1}, b_{2}\right)= \begin{cases}V_{d 1}\left(u ; b_{1}, b_{2}\right), & 0 \leq u<b_{1}, \\
V_{d 2}\left(u ; b_{1}, b_{2}\right), & b_{1} \leq u \leq b_{2} .\end{cases} \\
& V_{r}\left(u ; b_{1}, b_{2}\right)= \begin{cases}V_{r 1}\left(u ; b_{1}, b_{2}\right), & 0 \leq u<b_{1}, \\
V_{r 2}\left(u ; b_{1}, b_{2}\right), & b_{1} \leq u \leq b_{2} .\end{cases}
\end{aligned}
$$

Then, we have

$$
V\left(u ; b_{1}, b_{2}\right)= \begin{cases}V_{1^{*}}\left(u ; b_{1}, b_{2}\right)=V_{d 1}\left(u ; b_{1}, b_{2}\right)+V_{r 1}\left(u ; b_{1}, b_{2}\right), & 0 \leq u<b_{1}, \\ V_{2^{*}}\left(u ; b_{1}, b_{2}\right)=V_{d 2}\left(u ; b_{1}, b_{2}\right)+V_{r 2}\left(u ; b_{1}, b_{2}\right), & b_{1} \leq u \leq b_{2} .\end{cases}
$$

In the following, we first derive the integro-differential equations and boundary conditions satisfied by $V_{d}\left(u ; b_{1}, b_{2}\right)$ and $V_{r}\left(u ; b_{1}, b_{2}\right)$.

Theorem 3.1 Assume that $V_{d}\left(u ; b_{1}, b_{2}\right)$ is continuously differentiable in $u$ on $\left(0, b_{1}\right) \cup\left(b_{1}, b_{2}\right)$. Then, $V_{d}\left(u ; b_{1}, b_{2}\right)$ satisfies the following integro-differential equations, when $0<u<b_{1}$,

$$
\mu \frac{\partial V_{d 1}\left(u ; b_{1}, b_{2}\right)}{\partial u}-(\lambda+\delta) V_{d 1}\left(u ; b_{1}, b_{2}\right)+\lambda \int_{0}^{u} V_{d 1}\left(u-y ; b_{1}, b_{2}\right) p(y) \mathrm{d} y=0,
$$

and, when $b_{1}<u<b_{2}$,

$$
\begin{aligned}
& \alpha+(\mu-\alpha) \frac{\partial V_{d 2}\left(u ; b_{1}, b_{2}\right)}{\partial u}-(\lambda+\delta) V_{d 2}\left(u ; b_{1}, b_{2}\right) \\
& +\lambda\left[\int_{0}^{u-b_{1}} V_{d 2}\left(u-y ; b_{1}, b_{2}\right) p(y) \mathrm{d} y+\int_{u-b_{1}}^{u} V_{d 1}\left(u-y ; b_{1}, b_{2}\right) p(y) \mathrm{d} y\right]=0,
\end{aligned}
$$

with boundary conditions

$$
V_{d 1}\left(b_{1}-; b_{1}, b_{2}\right)=V_{d 2}\left(b_{1}+; b_{1}, b_{2}\right),
$$




$$
\begin{gathered}
\left.\frac{\partial V_{d 2}\left(u ; b_{1}, b_{2}\right)}{\partial u}\right|_{u=b_{2}}=0, \\
\left.\mu \frac{\partial V_{d 1}\left(u ; b_{1}, b_{2}\right)}{\partial u}\right|_{u=b_{1}-}=\alpha+\left.(\mu-\alpha) \frac{\partial V_{d 2}\left(u ; b_{1}, b_{2}\right)}{\partial u}\right|_{u=b_{1}+} .
\end{gathered}
$$

Proof. When $0 \leq u<b_{1}$, consider $t>0$ such that the modified surplus can not reach level $b_{1}$ by time $t$, i.e. $u+\mu t<b_{1}$. In view of the strong Markov property of the surplus process $\{\tilde{U}, t \geq 0\}$, we have

$$
\begin{aligned}
V_{d 1}\left(u ; b_{1}, b_{2}\right) & =E_{u}\left[\int_{t}^{T} \mathrm{e}^{-\delta s} \mathrm{~d} D_{1}(t)\right]+o(t) \\
& =E_{u}\left[\int_{0}^{T-t} \mathrm{e}^{-\delta(s+t)} \mathrm{d} D_{1}(t+s)\right]+o(t) \\
& =\mathrm{e}^{-\delta t} E_{u}\left[\int_{0}^{T} \mathrm{e}^{-\delta s} \mathrm{~d} D_{1}(s) \circ \theta_{t}\right]+o(t) \\
& =\mathrm{e}^{-\delta t} E_{u}\left\{E_{\tilde{U}_{t}}\left[\int_{0}^{T} \mathrm{e}^{-\delta s} \mathrm{~d} D_{1}(s)\right]\right\}+o(t) \\
& =\mathrm{e}^{-\delta t} E_{u}\left[V_{d 1}\left(\tilde{U}_{t} ; b_{1}, b_{2}\right)\right]+o(t),
\end{aligned}
$$

where $\theta_{t}$ is the shift operator. By conditioning on the time and amount of the first claim and whether the claim causes ruin or not, and using (3.6), we get

$$
V_{d 1}\left(u ; b_{1}, b_{2}\right)=\mathrm{e}^{-\delta t}(1-\lambda t) V_{d 1}\left(u+\mu t ; b_{1}, b_{2}\right)+\mathrm{e}^{-\delta t} \lambda t \int_{0}^{u+\mu t} V_{d 1}\left(u+\mu t-y ; b_{1}, b_{2}\right) p(y) \mathrm{d} y+o(t) .
$$

By Taylor's expansion,

$$
V_{d 1}\left(u+\mu t ; b_{1}, b_{2}\right)=V_{d 1}\left(u ; b_{1}, b_{2}\right)+\mu t \frac{\partial V_{d 1}\left(u ; b_{1}, b_{2}\right)}{\partial u}+o(t) .
$$

Substituting the above expressions into (3.7), and dividing both sides of (3.7) by $t$ and letting $t \rightarrow 0$, we can get (3.1).

When $b_{1}<u<b_{2}$, we still consider a small time interval [0,t], with $t(>0)$ being sufficiently small so that the modified surplus will not reach $b_{2}$ in the time interval. In view of the strong Markov property of the surplus process $\left\{\tilde{U}_{t}, t \geq 0\right\}$, we have

$$
\begin{aligned}
V_{d 2}\left(u ; b_{1}, b_{2}\right) & =\alpha t+E_{u}\left[\int_{t}^{T} \mathrm{e}^{-\delta s} \mathrm{~d} D_{1}(t)\right]+o(t) \\
& =\alpha t+\mathrm{e}^{-\delta t} E_{u}\left[V_{d 2}\left(\tilde{U}_{t} ; b_{1}, b_{2}\right)\right]+o(t) .
\end{aligned}
$$

By conditioning on the time and amount of the first claim and whether the claim causes ruin or not, and using (3.8), we get

$$
\begin{aligned}
V_{d 2}\left(u ; b_{1}, b_{2}\right)= & \alpha t+\mathrm{e}^{-\delta t}(1-\lambda t) V_{d 2}\left(u+(\mu-\alpha) t ; b_{1}, b_{2}\right) \\
& +\mathrm{e}^{-\delta t} \lambda t\left[\int_{0}^{u+(\mu-\alpha) t-b_{1}} V_{d 2}\left(u+(\mu-\alpha) t-y ; b_{1}, b_{2}\right) p(y) \mathrm{d} y\right. \\
& \left.+\int_{u+(\mu-\alpha) t-b_{1}}^{u+(\mu-\alpha) t} V_{d 1}\left(u+(\mu-\alpha) t-y ; b_{1}, b_{2}\right) p(y) \mathrm{d} y\right]+o(t) .
\end{aligned}
$$

By Taylor's expansion,

$$
V_{d 2}\left(u+(\mu-\alpha) t ; b_{1}, b_{2}\right)=V_{d 2}\left(u ; b_{1}, b_{2}\right)+(\mu-\alpha) t \frac{\partial V_{d 2}\left(u ; b_{1}, b_{2}\right)}{\partial u}+o(t) .
$$

Substituting the above expressions into (3.9), and dividing both sides of (3.9) by $t$ and letting $t \rightarrow 0$, we can get (3.2).

Next we prove the condition (3.3). It follows from 


$$
\mathrm{e}^{\frac{-(\delta+\lambda) \epsilon}{\mu}} V_{d 2}\left(b_{1} ; b_{1}, b_{2}\right) \leq V_{d 1}\left(b_{1}-\epsilon ; b_{1}, b_{2}\right) \leq V_{d 2}\left(b_{1} ; b_{1}, b_{2}\right), \quad 0<\epsilon \leq b_{1},
$$

let $\epsilon \downarrow 0$, we have

$$
V_{d 1}\left(b_{1}-; b_{1}, b_{2}\right)=V_{d 2}\left(b_{1} ; b_{1}, b_{2}\right)
$$

Similarly,

$$
\mathrm{e}^{\frac{-(\delta+\lambda) \epsilon}{\mu}} V_{d 2}\left(b_{1}+\epsilon ; b_{1}, b_{2}\right) \leq V_{d 2}\left(b_{1} ; b_{1}, b_{2}\right) \leq V_{d 2}\left(b_{1}+\epsilon ; b_{1}, b_{2}\right), 0<\epsilon \leq b_{2}-b_{1},
$$

let $\epsilon \downarrow 0$, we obtain

$$
V_{d 2}\left(b_{1}+; b_{1}, b_{2}\right)=V_{d 2}\left(b_{1} ; b_{1}, b_{2}\right)
$$

So we get (3.3).

Furthermore, when the initial surplus is $b_{2}$, we can mimic the derivation of (3.9) to obtain

$$
\begin{aligned}
V_{d 2}\left(b_{2} ; b_{1}, b_{2}\right)= & \alpha t+\mathrm{e}^{-\delta t}(1-\lambda t) V_{d 2}\left(b_{2} ; b_{1}, b_{2}\right) \\
& +\mathrm{e}^{-\delta t} \lambda t\left[\int_{0}^{b_{2}-b_{1}} V_{d 2}\left(b_{2}-y ; b_{1}, b_{2}\right) p(y) \mathrm{d} y\right. \\
& \left.+\int_{b_{2}-b_{1}}^{b_{2}} V_{d 1}\left(b_{2}-y ; b_{1}, b_{2}\right) p(y) \mathrm{d} y\right]+o(t) .
\end{aligned}
$$

Dividing both sides of (3.10) by $t$ and letting $t \rightarrow 0$, we can obtain

$$
(\delta+\lambda) V_{d 2}\left(b_{2} ; b_{1}, b_{2}\right)=\alpha+\lambda\left[\int_{0}^{b_{2}-b_{1}} V_{d 2}\left(b_{2}-y ; b_{1}, b_{2}\right) p(y) \mathrm{d} y+\int_{b_{2}-b_{1}}^{b_{2}} V_{d 1}\left(b_{2}-y ; b_{1}, b_{2}\right) p(y) \mathrm{d} y\right] .
$$

Letting $u \uparrow b_{2}$ in (3.2) and comparing it to (3.11), we obtain

$$
\left.\frac{\partial V_{d 2}\left(u ; b_{1}, b_{2}\right)}{\partial u}\right|_{u=b_{2}-}=0 .
$$

When $u>b_{2}$, we have

$$
V_{d}\left(u ; b_{1}, b_{2}\right)=V_{d 2}\left(b_{2} ; b_{1}, b_{2}\right)
$$

thus,

$$
\left.\frac{\partial V_{d}\left(u ; b_{1}, b_{2}\right)}{\partial u}\right|_{u=b_{2}+}=0
$$

So we get (3.4).

Finally, letting $u \uparrow b_{1}$ in (3.1) and $u \downarrow b_{1}$ in (3.2), we can get (3.5). This completes the proof of Theorem 3.1.

Remark 3.1 Letting $b_{2} \rightarrow \infty$ in Theorem 3.1, then (3.1) and (3.2) reduce, respectively, to (5.1) and (5.2) of [7].

Theorem 3.2 Assume that $V_{r}\left(u ; b_{1}, b_{2}\right)$ is continuously differentiable in $u$ on $\left(0, b_{1}\right) \cup\left(b_{1}, b_{2}\right)$. Then, $V_{r}\left(u ; b_{1}, b_{2}\right)$ satisfies the following integro-differential equations, when $0<u<b_{1}$,

$$
\mu \frac{\partial V_{r 1}\left(u ; b_{1}, b_{2}\right)}{\partial u}-(\lambda+\delta) V_{r 1}\left(u ; b_{1}, b_{2}\right)+\lambda \int_{0}^{u} V_{r 1}\left(u-y ; b_{1}, b_{2}\right) p(y) \mathrm{d} y=0,
$$

and, when $b_{1}<u<b_{2}$,

$$
\begin{aligned}
& (\mu-\alpha) \frac{\partial V_{r 2}\left(u ; b_{1}, b_{2}\right)}{\partial u}-(\lambda+\delta) V_{r 2}\left(u ; b_{1}, b_{2}\right) \\
& +\lambda\left[\int_{0}^{u-b_{1}} V_{r 2}\left(u-y ; b_{1}, b_{2}\right) p(y) \mathrm{d} y+\int_{u-b_{1}}^{u} V_{r 1}\left(u-y ; b_{1}, b_{2}\right) p(y) \mathrm{d} y\right]=0,
\end{aligned}
$$

with boundary conditions 


$$
\begin{gathered}
V_{r 1}\left(b_{1}-; b_{1}, b_{2}\right)=V_{r 2}\left(b_{1}+; b_{1}, b_{2}\right), \\
\left.\frac{\partial V_{r 2}\left(u ; b_{1}, b_{2}\right)}{\partial u}\right|_{u=b_{2}}=1, \\
\left.\mu \frac{\partial V_{d 1}\left(u ; b_{1}, b_{2}\right)}{\partial u}\right|_{u=b_{1}-}=\left.(\mu-\alpha) \frac{\partial V_{d 2}\left(u ; b_{1}, b_{2}\right)}{\partial u}\right|_{u=b_{1}+} .
\end{gathered}
$$

Proof. In view of the strong Markov property of the surplus process $\left\{\tilde{U}_{t}, t \geq 0\right\}$, we have

$$
V_{r}\left(u ; b_{1}, b_{2}\right)=\mathrm{e}^{-\delta t} E_{u}\left[V_{r}\left(\tilde{U}_{t} ; b_{1}, b_{2}\right)\right]+o(t) .
$$

When $0<u<b_{1}$, we consider a small time interval $[0, t]$, with $t>0$ being sufficiently small so that the modified surplus will not reach $b_{1}$ in the interval. By conditioning on the time and amount of the first claim and whether the claim causes ruin or not, and using (3.17), we get

$$
\begin{aligned}
V_{r 1}\left(u ; b_{1}, b_{2}\right)= & \mathrm{e}^{-\delta t}(1-\lambda t) V_{r 1}\left(u+\mu t ; b_{1}, b_{2}\right) \\
& +\mathrm{e}^{-\delta t} \lambda t \int_{0}^{u+\mu t} V_{r 1}\left(u+\mu t-y ; b_{1}, b_{2}\right) p(y) \mathrm{d} y+o(t) .
\end{aligned}
$$

By Taylor's expansion,

$$
V_{r 1}\left(u+\mu t ; b_{1}, b_{2}\right)=V_{r 1}\left(u ; b_{1}, b_{2}\right)+\mu t \frac{\partial V_{r 1}\left(u ; b_{1}, b_{2}\right)}{\partial u}+o(t) .
$$

Substituting the above expression into (3.18), and dividing both sides of (3.18) by $t$ and letting $t \rightarrow 0$, we can get (12).

When $b_{1}<u<b_{2}$, we still consider a small time interval [0,t], with $t>0$ being sufficiently small so that the modified surplus will not reach $b_{2}$ in the interval. Similar to the derivation of (3.12), we can obtain Equation (3.13).

The condition (3.14) can be obtained similar to (3.3).

When the initial surplus is $b_{2}$, we have

$$
\begin{aligned}
V_{r 2}\left(b_{2} ; b_{1}, b_{2}\right)= & \mathrm{e}^{-\delta t}(1-\lambda t)\left[(\mu-\alpha) t+V_{r 2}\left(b_{2} ; b_{1}, b_{2}\right)\right] \\
& +\mathrm{e}^{-\delta t} \lambda t\left[\int_{0}^{b_{2}-b_{1}} V_{r 2}\left(b_{2}-y ; b_{1}, b_{2}\right) p(y) \mathrm{d} y\right. \\
& \left.+\int_{b_{2}-b_{1}}^{b_{2}} V_{r 1}\left(b_{2}-y ; b_{1}, b_{2}\right) p(y) \mathrm{d} y\right]+o(t) .
\end{aligned}
$$

Dividing both sides of (3.19) by $t$ and letting $t \rightarrow 0$, we can obtain

$$
\begin{aligned}
(\delta+\lambda) V_{r 2}\left(b_{2} ; b_{1}, b_{2}\right)= & (\mu-\alpha)+\lambda\left[\int_{0}^{b_{2}-b_{1}} V_{r 2}\left(b_{2}-y ; b_{1}, b_{2}\right) p(y) \mathrm{d} y\right. \\
& \left.+\int_{b_{2}-b_{1}}^{b_{2}} V_{r 1}\left(b_{2}-y ; b_{1}, b_{2}\right) p(y) \mathrm{d} y\right] .
\end{aligned}
$$

Letting $u \uparrow b_{2}$ in (3.13) and comparing it to (3.20), we obtain

$$
\left.\frac{\partial V_{r 2}\left(u ; b_{1}, b_{2}\right)}{\partial u}\right|_{u=b_{2}-}=1 .
$$

When $u>b_{2}$, we have

$$
V_{r}\left(u ; b_{1}, b_{2}\right)=u-b_{2}+V_{r 2}\left(b_{2} ; b_{1}, b_{2}\right)
$$

thus,

$$
\left.\frac{\partial V_{r}\left(u ; b_{1}, b_{2}\right)}{\partial u}\right|_{u=b_{2}+}=1 .
$$


So we get (3.15).

Finally, letting $u \uparrow b_{1}$ in (3.12) and $u \downarrow b_{1}$ in (3.13), we can get (3.16). This completes the proof of Theorem 3.2.

According to the definition of $V\left(u ; b_{1}, b_{2}\right)$, from Theorems 3.1 and 3.2, we can lead to the integro-differential equations and the boundary conditions satisfied by $V\left(u ; b_{1}, b_{2}\right)$.

Theorem 3.3 Assume that $V\left(u ; b_{1}, b_{2}\right)$ is continuously differentiable in $u$ on $\left(0, b_{1}\right) \cup\left(b_{1}, b_{2}\right)$. Then, $V\left(u ; b_{1}, b_{2}\right)$ satisfies the following integro-differential equations, when $0<u<b_{1}$,

$$
\mu \frac{\partial V_{1 *}\left(u ; b_{1}, b_{2}\right)}{\partial u}-(\lambda+\delta) V_{1 *}\left(u ; b_{1}, b_{2}\right)+\lambda \int_{0}^{u} V_{1 *}\left(u-y ; b_{1}, b_{2}\right) p(y) \mathrm{d} y=0,
$$

and, when $b_{1}<u<b_{2}$,

$$
\begin{aligned}
& \alpha+(\mu-\alpha) \frac{\partial V_{2 *}\left(u ; b_{1}, b_{2}\right)}{\partial u}-(\lambda+\delta) V_{2 *}\left(u ; b_{1}, b_{2}\right)+\lambda\left[\int_{0}^{u-b_{1}} V_{2 *}\left(u-y ; b_{1}, b_{2}\right) p(y) \mathrm{d} y\right. \\
& \left.+\int_{u-b_{1}}^{u} V_{1 *}\left(u-y ; b_{1}, b_{2}\right) p(y) \mathrm{d} y\right]=0,
\end{aligned}
$$

with boundary conditions

$$
\begin{gathered}
V_{1 *}\left(b_{1}-; b_{1}, b_{2}\right)=V_{2 *}\left(b_{1}+; b_{1}, b_{2}\right), \\
\left.\frac{\partial V_{2 *}\left(u ; b_{1}, b_{2}\right)}{\partial u}\right|_{u=b_{2}}=1, \\
\left.\mu \frac{\partial V_{1 *}\left(u ; b_{1}, b_{2}\right)}{\partial u}\right|_{u=b_{1-}}=\alpha+\left.(\mu-\alpha) \frac{\partial V_{2 *}\left(u ; b_{1}, b_{2}\right)}{\partial u}\right|_{u=b_{1}+} .
\end{gathered}
$$

Example 3.1. Now we assume that the individual claim amounts are exponentially distributed with mean $1 / \beta$, i.e.

$$
p(y)=\beta \mathrm{e}^{-\beta y}, \quad y>0 .
$$

Then, we have

$$
p^{\prime}(y)+\beta p(y)=0 .
$$

Applying the operator $(\mathrm{d} / \mathrm{d} u+\beta)$ on (3.21) and (3.22) respectively, and using (3.26) and rearranging them, we get

$$
\mu \frac{\partial V_{1 *}^{2}\left(u ; b_{1}, b_{2}\right)}{\partial u^{2}}+(\beta \mu-\lambda-\delta) \frac{\partial V_{1 *}\left(u ; b_{1}, b_{2}\right)}{\partial u}-\beta \delta V_{1 *}\left(u ; b_{1}, b_{2}\right)=0,
$$

for $0<u<b_{1}$, and for $b_{1}<u<b_{2}$

$$
(\mu-\alpha) \frac{\partial V_{2 *}^{2}\left(u ; b_{1}, b_{2}\right)}{\partial u^{2}}+[\beta(\mu-\alpha)-\lambda-\delta] \frac{\partial V_{2 *}\left(u ; b_{1}, b_{2}\right)}{\partial u}-\beta \delta V_{2 *}\left(u ; b_{1}, b_{2}\right)+\beta \alpha=0 .
$$

We can obtain the solutions of Equation (3.27) as follows

$$
V_{1^{*}}\left(u ; b_{1}, b_{2}\right)=A \mathrm{e}^{r u}+B \mathrm{e}^{s u}, \quad 0 \leq u \leq b_{1},
$$

with the coefficients $A$ and $B$ being independent of $u$, and $r$ and $s$ being the roots of the characteristic equation

$$
\mu \xi^{2}+(\beta \mu-\lambda-\delta) \xi-\beta \delta=0 .
$$

We let $r$ denote the positive root and $s$ the negative root, i.e.

$$
r=\frac{-(\beta \mu-\lambda-\delta)+\sqrt{(\beta \mu-\lambda-\delta)^{2}+4 \mu \beta \delta}}{2 \mu},
$$




$$
s=\frac{-(\beta \mu-\lambda-\delta)-\sqrt{(\beta \mu-\lambda-\delta)^{2}+4 \mu \beta \delta}}{2 \mu} .
$$

Substituting (3.29) in Equation (3.21) and equating the coefficient of $\mathrm{e}^{-\beta u}$ with 0 , we have

$$
\lambda \beta\left(\frac{A}{r+\beta}+\frac{B}{s+\beta}\right)=0 .
$$

From (3.29) and (3.30), we can rewrite

$$
V_{1 *}\left(u ; b_{1}, b_{2}\right)=\gamma\left[(r+\beta) \mathrm{e}^{r u}-(\beta+s) \mathrm{e}^{s u}\right], \quad 0<u<b_{1},
$$

where $\gamma$ dose not depend on $u$. A particular solution of (3.28) is $\alpha / \delta$. Hence, the solutions of Equation (3.28) are given by

$$
V_{2 *}\left(u ; b_{1}, b_{2}\right)=\frac{\alpha}{\delta}+C \mathrm{e}^{v u}+G \mathrm{e}^{w u}, \quad b_{1}<u \leq b_{2},
$$

where the coefficients $C$ and $G$ are independent of $u$, and $w<0$ and $v>0$ are the roots of the characteristic equation

$$
(\mu-\alpha) \xi^{2}+[\beta(\mu-\alpha)-\lambda-\delta] \xi-\delta \beta=0
$$

namely,

$$
\begin{aligned}
& v=\frac{-[\beta(\mu-\alpha)-\lambda-\delta]+\sqrt{[\beta(\mu-\alpha)-\lambda-\delta]^{2}+4(\mu-\alpha) \beta \delta}}{2(\mu-\alpha)}, \\
& w=\frac{-[\beta(\mu-\alpha)-\lambda-\delta]-\sqrt{[\beta(\mu-\alpha)-\lambda-\delta]^{2}+4(\mu-\alpha) \beta \delta}}{2(\mu-\alpha)} .
\end{aligned}
$$

From (3.31) and (3.32), we observe that the convolution integral in Equation (3.22) is

$$
\begin{aligned}
& \int_{0}^{b_{1}} V_{1 *}\left(y ; b_{1}, b_{2}\right) p(u-y) \mathrm{d} y+\int_{b_{1}}^{u} V_{2 *}\left(y ; b_{1}, b_{2}\right) p(u-y) \mathrm{d} y \\
& =\gamma \int_{0}^{b_{1}}\left[(r+\beta) \mathrm{e}^{r y}-(s+\beta) \mathrm{e}^{s y}\right] \beta \mathrm{e}^{-\beta(u-y)} \mathrm{d} y+\int_{b_{1}}^{u}\left(\frac{\alpha}{\delta}+C \mathrm{e}^{v y}+G \mathrm{e}^{w y}\right) \beta \mathrm{e}^{-\beta(u-y)} \mathrm{d} y \\
& =\beta \mathrm{e}^{-\beta u}\left\{\gamma\left[\mathrm{e}^{(r+\beta) b_{1}}-\mathrm{e}^{(s+\beta) b_{1}}\right]+\frac{\alpha}{\delta \beta}\left(\mathrm{e}^{\beta u}-\mathrm{e}^{\beta b_{1}}\right)+\frac{C}{\beta+v}\left[\mathrm{e}^{(\beta+v) u}-\mathrm{e}^{(\beta+v) b_{1}}\right]+\frac{G}{\beta+w}\left[\mathrm{e}^{(\beta+w) u}-\mathrm{e}^{(\beta+w) b_{1}}\right]\right\} .
\end{aligned}
$$

By setting the coefficient of $\mathrm{e}^{-\beta u}$ to 0 , we have

$$
\gamma\left(\mathrm{e}^{r b_{1}}-\mathrm{e}^{s b_{1}}\right)=\frac{\alpha}{\delta \beta}+\frac{C}{\beta+v} \mathrm{e}^{v b_{1}}+\frac{G}{\beta+w} \mathrm{e}^{w b_{1}} .
$$

From (23) and (24), we have the conditions

$$
\gamma\left[(r+\beta) \mathrm{e}^{r b_{1}}-(s+\beta) \mathrm{e}^{s b_{1}}\right]=\frac{\alpha}{\delta}+C \mathrm{e}^{v b_{1}}+G \mathrm{e}^{w b_{1}},
$$

and

$$
C v \mathrm{e}^{v b_{2}}+G w \mathrm{e}^{w b_{2}}=1
$$

It follows from (33) and (34) that

$$
C=\frac{\beta+v}{w-v}\left\{\gamma\left[(w-r) \mathrm{e}^{r b_{1}}-(w-s) \mathrm{e}^{s b_{1}}\right]-\frac{\alpha w}{\delta \beta}\right\} \mathrm{e}^{-v b_{1}} .
$$




$$
G=\frac{\beta+w}{v-w}\left\{\gamma\left[(v-r) \mathrm{e}^{r b_{1}}-(v-s) \mathrm{e}^{s b_{1}}\right]-\frac{\alpha v}{\delta \beta}\right\} \mathrm{e}^{-w b_{1}} .
$$

Substitution of (3.36) and (3.37) into (3.35), thus we get the closed-form solution of $\gamma$ as follows,

$$
\gamma=\frac{\alpha w v\left[(\beta+w) \mathrm{e}^{w\left(b_{2}-b_{1}\right)}+(\beta+v) \mathrm{e}^{v\left(b_{2}-b_{1}\right)}\right]+\delta \beta(w-v)}{E},
$$

where

$$
\begin{aligned}
E= & \delta \beta\left\{v(\beta+v)\left[(w-r) \mathrm{e}^{r b_{1}}-(w-s) \mathrm{e}^{s b_{1}}\right] \mathrm{e}^{v\left(b_{2}-b_{1}\right)}\right. \\
& \left.-w(\beta+w)\left[(v-r) \mathrm{e}^{r b_{1}}-(v-s) \mathrm{e}^{s b_{1}}\right] \mathrm{e}^{w\left(b_{2}-b_{1}\right)}\right\} .
\end{aligned}
$$

We can get $C$ and $G$ by substituting $\gamma$ into (3.36) and (3.37).

Hence

$$
V_{1 *}\left(u ; b_{1}, b_{2}\right)=\gamma\left[(r+\beta) \mathrm{e}^{r u}-(s+\beta) \mathrm{e}^{s u}\right], \text { if } 0 \leq u<b_{1},
$$

and

$$
\begin{aligned}
V_{2 *}\left(u ; b_{1}, b_{2}\right)= & \frac{\alpha}{\delta}+\frac{\beta+v}{w-v}\left\{\gamma\left[(w-r) \mathrm{e}^{r b_{1}}-(w-s) \mathrm{e}^{s b_{1}}\right]-\frac{\alpha w}{\delta \beta}\right\} \mathrm{e}^{v\left(u-b_{1}\right)} \\
& +\frac{\beta+w}{v-w}\left\{\gamma\left[(v-r) \mathrm{e}^{r b_{1}}-(v-s) \mathrm{e}^{s b_{1}}\right]-\frac{\alpha v}{\delta \beta}\right\} \mathrm{e}^{w\left(u-b_{1}\right)}, \quad \text { if } b_{1} \leq u \leq b_{2} .
\end{aligned}
$$

Remark 3.2 Let us compare our results with known results.

1) When $b_{1}=b_{2}, \alpha=\mu$, the hybrid dividend becomes a barrier dividend strategy, the condition (3.25) is the same as (3.24), from (3.31) and (3.24), we have

$$
\gamma=\frac{1}{(\beta+r) r \mathrm{e}^{r b_{1}}-(\beta+s) s \mathrm{e}^{s b_{1}}}
$$

which agrees with formula (7.8) in [2].

2) Letting $b_{2} \rightarrow \infty$, the hybrid dividend strategy becomes a threshold dividend strategy, we get

$$
\lim _{b_{2} \rightarrow \infty} \gamma=\frac{\alpha w}{\delta \beta} \frac{1}{(w-r) \mathrm{e}^{r b_{1}}-(w-s) \mathrm{e}^{s b_{1}}} .
$$

From (3.36), (3.37) and (3.42), we have

$$
\begin{gathered}
\lim _{b_{2} \rightarrow \infty} G=\frac{\alpha(\beta+w)}{\delta \beta} \frac{r \mathrm{e}^{r b_{1}}-s \mathrm{e}^{s b_{1}}}{(w-r) \mathrm{e}^{r b_{1}}-(w-s) \mathrm{e}^{s b_{1}}} \mathrm{e}^{-w b_{1}} . \\
\lim _{b_{2} \rightarrow \infty} C=0 .
\end{gathered}
$$

It follows from (3.40) to (3.44) that

$$
\lim _{b_{2} \rightarrow \infty} V_{1 *}\left(u ; b_{1}, b_{2}\right)=\frac{\alpha w}{\delta \beta} \frac{(\beta+r) \mathrm{e}^{r u}-(\beta-s) \mathrm{e}^{s u}}{(w-r) \mathrm{e}^{r b_{1}}-(w-s) \mathrm{e}^{s b_{1}}}, \text { if } 0 \leq u \leq b_{1},
$$

and

$$
\begin{aligned}
\lim _{b_{2} \rightarrow \infty} V_{2 *}\left(u ; b_{1}, b_{2}\right) & =\frac{\alpha}{\delta}+\frac{\alpha(\beta+w)}{\delta \beta} \frac{r \mathrm{e}^{r b_{1}}-s \mathrm{e}^{s b_{1}}}{(w-r) \mathrm{e}^{r b_{1}}-(w-s) \mathrm{e}^{s b_{1}}} \mathrm{e}^{w\left(u-b_{1}\right)} \\
& =\frac{\alpha}{\delta}\left[1-\mathrm{e}^{w\left(u-b_{1}\right)}\right]+\lim _{b_{2} \rightarrow \infty} V_{1 *}\left(b_{1} ; b_{1}, b_{2}\right) \mathrm{e}^{w\left(u-b_{1}\right)}, \quad \text { if } b_{1} \leq u,
\end{aligned}
$$

which are (6.14) and (6.15) in [7]. 


\section{The Moment-Generating Function}

In this section, we study the moment-generating function $M\left(u, z ; b_{1}, b_{2}\right)$ which has been discussed in various models, for example, see [8] [19]. We can analyze the moments of $D$ through $M\left(u, z ; b_{1}, b_{2}\right)$. Since $M\left(u, z ; b_{1}, b_{2}\right)$ has different paths for $0 \leq u<b_{1}$ and $b_{1} \leq u \leq b_{2}$, we define

$$
M\left(u, z ; b_{1}, b_{2}\right)= \begin{cases}M_{1}\left(u, z ; b_{1}, b_{2}\right), & 0 \leq u<b_{1}, \\ M_{2}\left(u, z ; b_{1}, b_{2}\right), & b_{1} \leq u \leq b_{2} .\end{cases}
$$

We first derive the integro-differential equations and boundary conditions for $M\left(u, z ; b_{1}, b_{2}\right)$.

Theorem 4.1 Assume that $M\left(u, z ; b_{1}, b_{2}\right)$ is continuously differentiable in $u$ on $\left(0, b_{1}\right) \cup\left(b_{1}, b_{2}\right)$ and in $y \geq 0$. Then, $M\left(u, z ; b_{1}, b_{2}\right)$ satisfies the following integro-differential equations, when $0<u<b_{1}$,

$$
\begin{aligned}
\mu \frac{\partial M_{1}\left(u, z ; b_{1}, b_{2}\right)}{\partial u}= & \delta z \frac{\partial M_{1}\left(u, z ; b_{1}, b_{2}\right)}{\partial z}+\lambda M_{1}\left(u, z ; b_{1}, b_{2}\right) \\
& -\lambda\left[\int_{0}^{u} M_{1}\left(u-y, z ; b_{1}, b_{2}\right) p(y) \mathrm{d} y+1-P(u)\right],
\end{aligned}
$$

and, when $b_{1}<u<b_{2}$,

$$
\begin{aligned}
(\mu-\alpha) \frac{\partial M_{2}\left(u, z ; b_{1}, b_{2}\right)}{\partial u}= & \delta z \frac{\partial M_{2}\left(u, z ; b_{1}, b_{2}\right)}{\partial z}+(\lambda-z \alpha) M_{2}\left(u, z ; b_{1}, b_{2}\right) \\
& -\lambda\left[\int_{0}^{u-b_{1}} M_{2}\left(u-y, z ; b_{1}, b_{2}\right) p(y) \mathrm{d} y\right. \\
& \left.+\int_{u-b_{1}}^{u} M_{1}\left(u-y, z ; b_{1}, b_{2}\right) p(y) \mathrm{d} y+1-P(u)\right],
\end{aligned}
$$

with boundary conditions

$$
\begin{gathered}
M_{1}\left(b_{1}-, z ; b_{1}, b_{2}\right)=M_{2}\left(b_{1}+, z ; b_{1}, b_{2}\right), \\
\left.\frac{\partial M_{2}\left(u, z ; b_{1}, b_{2}\right)}{\partial u}\right|_{u=b_{2}-}=z M_{2}\left(b_{2}, z ; b_{1}, b_{2}\right), \\
\left.\mu \frac{\partial M_{1}\left(u, z ; b_{1}, b_{2}\right)}{\partial u}\right|_{u=b_{1}-}=\left.(\mu-\alpha) \frac{\partial M_{2}\left(u, z ; b_{1}, b_{2}\right)}{\partial u}\right|_{u=b_{1}+}+z \alpha M_{2}\left(b_{1}, z ; b_{1}, b_{2}\right) .
\end{gathered}
$$

Proof. In view of the strong Markov property of the surplus process $\left\{\tilde{U}_{t}, t \geq 0\right\}$, we have

$$
M_{1}\left(u, z ; b_{1}, b_{2}\right)=E\left[M_{1}\left(\tilde{U}_{t}, z \mathrm{e}^{-\delta t} ; b_{1}, b_{2}\right)\right]+o(t),
$$

when $0 \leq u<b_{1}$, consider $t>0$ being sufficiently small so that the modified surplus can not reach level $b_{1}$ by time $t$. By conditioning on the time and amount of the first claim and whether the claim causes ruin or not, and using (4.6), we get

$$
\begin{aligned}
M_{1}\left(u, z ; b_{1}, b_{2}\right)= & (1-\lambda t) M_{1}\left(u+\mu t, \mathrm{ze}^{-\delta t} ; b_{1}, b_{2}\right) \\
& +\lambda t\left[\int_{0}^{u+\mu t} M_{1}\left(u+\mu t-y, z \mathrm{e}^{-\delta t} ; b_{1}, b_{2}\right) p(y) \mathrm{d} y\right] \\
& +\lambda t[1-P(u+\mu t)]+o(t) .
\end{aligned}
$$

By Taylor's expansion,

$$
M_{1}\left(u+\mu t, z \mathrm{e}^{-\delta t} ; b_{1}, b_{2}\right)=M_{1}\left(u, z ; b_{1}, b_{2}\right)+\mu t \frac{\partial M_{1}\left(u, z ; b_{1}, b_{2}\right)}{\partial u}-\delta z t \frac{\partial M_{1}\left(u, z ; b_{1}, b_{2}\right)}{\partial z}+o(t) .
$$

Substituting the above expression into (4.7), and dividing both sides of (4.7) by $t$ and letting $t \rightarrow 0$, we can get (4.1).

When $b_{1}<u<b_{2}$, we still consider a small time interval [0,t], with $t>0$ being sufficiently small so that 
the modified surplus will not reach $b_{2}$ in the interval. In view of the strong Markov property of the surplus process $\left\{\tilde{U}_{t}, t \geq 0\right\}$, we have

$$
M_{2}\left(u, z ; b_{1}, b_{2}\right)=\mathrm{e}^{z \alpha t} E_{u}\left[M_{2}\left(\tilde{U}_{t}, z \mathrm{e}^{-\delta t} ; b_{1}, b_{2}\right)\right]+o(t) .
$$

By conditioning on the time and amount of the first claim and whether the claim causes ruin or not, and using (4.8), we yield

$$
\begin{aligned}
M_{2}\left(u, z ; b_{1}, b_{2}\right)= & (1-\lambda t) \mathrm{e}^{z \alpha t} M_{2}\left(u+(\mu-\alpha) t, z \mathrm{e}^{-\delta t} ; b_{1}, b_{2}\right) \\
& +\lambda t \mathrm{e}^{z \alpha t}\left[\int_{0}^{u+(\mu-\alpha) t-b_{1}} M_{2}\left(u+(\mu-\alpha) t-y, z \mathrm{e}^{-\delta t} ; b_{1}, b_{2}\right) p(y) \mathrm{d} y\right. \\
& \left.+\int_{u+(\mu-\alpha) t-b_{1}}^{u+(\mu-\alpha) t} M_{1}\left(u+(\mu-\alpha) t-y, z \mathrm{e}^{-\delta t} ; b_{1}, b_{2}\right) p(y) \mathrm{d} y\right] \\
& +\lambda t \mathrm{e}^{z \alpha t}[1-P(u+(\mu-\alpha) t)]+o(t) .
\end{aligned}
$$

Since

$$
\begin{aligned}
& M_{2}\left(u+(\mu-\alpha) t, z \mathrm{e}^{-\delta t} ; b_{1}, b_{2}\right) \\
& =M_{2}\left(u, z ; b_{1}, b_{2}\right)+(\mu-\alpha) t \frac{\partial M_{2}\left(u, z ; b_{1}, b_{2}\right)}{\partial u}-\delta z t \frac{\partial M_{2}\left(u, z ; b_{1}, b_{2}\right)}{\partial z}+o(t),
\end{aligned}
$$

using the similar arguments as above, we get (4.2) from (4.9).

Next we prove the condition (4.3). For $0 \leq u<b_{1}$, let $\tau_{b_{1}}=\inf \left\{t: \tilde{U}_{t}=b_{1}, 0 \leq U_{0}<b_{1}\right\}$, and $t_{0}$ is the time that the modified surplus reaches $b_{1}$ for the first time from $0 \leq u<b_{1}$ with no claims, i.e. $u+\mu t_{0}=b_{1}$. Then $\tau_{b_{1}}$ is a stopping time, and by the strong Markov property, we have

$$
\begin{aligned}
M_{1}\left(u, z ; b_{1}, b_{2}\right) & =E_{u}\left[\mathrm{e}^{z D} I\left(\tau_{b_{1}}<T\right)\right]+E_{u}\left[\mathrm{e}^{z D} I\left(\tau_{b_{1}} \geq T\right)\right] \\
& =E_{u}\left[M_{2}\left(b_{1}, \mathrm{e}^{-\delta \tau_{b_{1}}} ; b_{1}, b_{2}\right) I\left(\tau_{b_{1}}<T\right)\right]+P\left(\tau_{b_{1}} \geq T\right) \\
& \leq M_{2}\left(b_{1}, z \mathrm{e}^{-\delta \tau_{b_{1}}} ; b_{1}, b_{2}\right)+P\left(\tau_{b_{1}} \geq T\right) .
\end{aligned}
$$

On the other hand, we have

$$
\begin{aligned}
M_{1}\left(u, z ; b_{1}, b_{2}\right) & \geq E_{u}\left[\mathrm{e}^{z D} I\left(\tau_{b_{1}}<T, \tau_{b_{1}}=t_{0}\right)\right]+E_{u}\left[\mathrm{e}^{z D} I\left(\tau_{b_{1}} \geq T\right)\right] \\
& =E_{u}\left[M_{2}\left(b_{1}, z \mathrm{e}^{-\delta \tau_{b_{1}}} ; b_{1}, b_{2}\right) I\left(\tau_{b_{1}}<T, \tau_{b_{1}}=t_{0}\right)\right]+P\left(\tau_{b_{1}} \geq T\right) \\
& \geq M_{2}\left(b_{1}, z \mathrm{e}^{-\delta t_{0}} ; b_{1}, b_{2}\right) P\left(T_{1}>t_{0}\right)+P\left(\tau_{b_{1}} \geq T\right),
\end{aligned}
$$

where $T_{1}$ is the first time that the claim happens. When $u \uparrow b_{1}, \tau_{b_{1}}$ and $t_{0}$ both go into zero, and $\lim _{u \uparrow b_{1}} P\left(\tau_{b_{1}} \geq T\right)=0$, letting $u \uparrow b_{1}$ in (4.10) and (4.11), we obtain

$$
M_{1}\left(b_{1}-, z ; b_{1}, b_{2}\right)=M_{2}\left(b_{1}, z ; b_{1}, b_{2}\right) .
$$

When $u=b_{1}$, we consider an infinitesimal time interval $[0, t]$, then

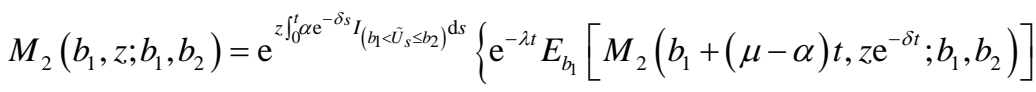

$$
\begin{aligned}
& \left.+E_{b_{1}}\left[M\left(\tilde{U}_{t}, \mathrm{ze}^{-\delta t} ; b_{1}, b_{2}\right) I(N(t)=1, T>t)\right]\right\}+O(t) .
\end{aligned}
$$

From this formula we get

$$
M_{2}\left(b_{1}, z ; b_{1}, b_{2}\right) \geq \mathrm{e}^{z \int_{0}^{t} \alpha \mathrm{e}^{-\delta s} I_{\left(b_{1}<\tilde{U}_{s} \leq b_{2}\right)^{\mathrm{ds}}}} \mathrm{e}^{-\lambda t} E_{b_{1}}\left[M_{2}\left(b_{1}+(\mu-\alpha) t, z \mathrm{e}^{-\delta t} ; b_{1}, b_{2}\right)\right],
$$

and 


$$
\begin{aligned}
M_{2}\left(b_{1}, z ; b_{1}, b_{2}\right) \leq & \mathrm{e}^{\left|z \int_{0}^{t} \alpha \mathrm{e}^{-\delta s} \mathrm{ds}\right|}\left\{\mathrm{e}^{-\lambda t} E_{b_{1}}\left[M_{2}\left(b_{1}+(\mu-\alpha) t, z \mathrm{e}^{-\delta t} ; b_{1}, b_{2}\right)\right]\right. \\
& \left.+E_{b_{1}}\left[M\left(\tilde{U}_{t}, z \mathrm{e}^{-\delta t} ; b_{1}, b_{2}\right) I(N(t)=1, T>t)\right]\right\}+|O(t)| .
\end{aligned}
$$

Let $t \downarrow 0$, we obtain

$$
M_{2}\left(b_{1}+, z ; b_{1}, b_{2}\right)=M_{2}\left(b_{1}, z ; b_{1}, b_{2}\right)
$$

So we obtain (4.3).

Furthermore, when the initial surplus is $b_{2}$, we can mimic the derivation of (4.9) to obtain

$$
\begin{aligned}
M_{2}\left(b_{2}, z ; b_{1}, b_{2}\right)= & (1-\lambda t) \mathrm{e}^{z(\mu-\alpha) t} \mathrm{e}^{z \alpha t} M_{2}\left(b_{2}, z \mathrm{e}^{-\delta t} ; b_{1}, b_{2}\right) \\
& +\lambda t \mathrm{e}^{z \alpha t}\left[\int_{0}^{b_{2}-b_{1}} M_{2}\left(b_{2}-y, z \mathrm{e}^{-\delta t} ; b_{1}, b_{2}\right) p(y) \mathrm{d} y\right. \\
& \left.+\int_{b_{2}-b_{1}}^{b_{2}} M_{1}\left(b_{2}-y, z e^{-\delta t} ; b_{1}, b_{2}\right) p(y) \mathrm{d} y\right] \\
& +\lambda t \mathrm{e}^{z \alpha t}\left[1-P\left(b_{2}\right)\right]+o(t)
\end{aligned}
$$

Using

$$
M_{2}\left(b_{2}, z \mathrm{e}^{-\delta t} ; b_{1}, b_{2}\right)=M_{2}\left(b_{2}, z ; b_{1}, b_{2}\right)-\delta z t \frac{\partial M_{2}\left(b_{2}, z ; b_{1}, b_{2}\right)}{\partial z}+o(t) .
$$

Substituting the above expression into (4.12), and dividing both sides of (4.12) by $t$ and letting $t \rightarrow 0$, we can obtain

$$
\begin{aligned}
\delta z \frac{\partial M_{2}\left(b_{2}, z ; b_{1}, b_{2}\right)}{\partial z}= & {[(\mu-\alpha) z+z \alpha-\lambda] M_{2}\left(b_{2}, z ; b_{1}, b_{2}\right) } \\
& +\lambda\left[\int_{0}^{b_{2}-b_{1}} M_{2}\left(b_{2}-y, z ; b_{1}, b_{2}\right) p(y) \mathrm{d} y\right. \\
& \left.+\int_{b_{2}-b_{1}}^{b_{2}} M_{1}\left(b_{2}-y, z ; b_{1}, b_{2}\right) p(y) \mathrm{d} y+1-P\left(b_{2}\right)\right] .
\end{aligned}
$$

Letting $u \uparrow b_{2}$ in (4.2) and comparing it to (4.13), we obtain

$$
\left.\frac{\partial M_{2}\left(u, z ; b_{1}, b_{2}\right)}{\partial u}\right|_{u=b_{2}-}=z M_{2}\left(b_{2}, z ; b_{1}, b_{2}\right) .
$$

Finally, letting $u \uparrow b_{1}$ in (4.1) and $u \downarrow b_{2}$ in (4.2), we can get (4.5). This completes the proof of Theorem 4.1.

Remark 4.1 1) In the case of $b_{1}=b_{2},(4.1)$ is corresponding to (3.1) of [20] by letting $a=0, \sigma=0$ and $\mu$ substitute $c$ there.

2) In the case of $b_{2} \rightarrow \infty,(4.1)$ and (4.2) are corresponding to (2.10) and (2.11) of [21] by letting $\sigma=0$, $r=0$ and $\mu$ substitute $c$ there.

By the definitions of $M\left(u, z ; b_{1}, b_{2}\right)$ and $V_{k}\left(u ; b_{1}, b_{2}\right)$, we obtain

$$
M\left(u, z ; b_{1}, b_{2}\right)=1+\sum_{k=1}^{\infty} \frac{z^{k}}{k !} V_{k}\left(u ; b_{1}, b_{2}\right)
$$

We denote

$$
V_{k}\left(u ; b_{1}, b_{2}\right)= \begin{cases}V_{k, 1}\left(u ; b_{1}, b_{2}\right), & 0 \leq u<b_{1}, \\ V_{k, 2}\left(u ; b_{1}, b_{2}\right), & b_{1} \leq u \leq b_{2} .\end{cases}
$$

Substituting (4.14) into (4.1) and (4.2) respectively and comparing the coefficients of $z^{k}$ yields the following integro-differential equations and corresponding boundary conditions.

Theorem 4.2 For each $k \geq 1$, we assume that $V_{k}\left(u ; b_{1}, b_{2}\right)$ is continuously differentiable in $u$ on 
$\left(0, b_{1}\right) \cup\left(b_{1}, b_{2}\right)$. Then, $V_{k}\left(u ; b_{1}, b_{2}\right)$ satisfies the following integro-differential equations:

$$
\mu \frac{\partial V_{k, 1}\left(u ; b_{1}, b_{2}\right)}{\partial u}=(k \delta+\lambda) V_{k, 1}\left(u ; b_{1}, b_{2}\right)-\lambda \int_{0}^{u} V_{k, 1}\left(u-y ; b_{1}, b_{2}\right) p(y) \mathrm{d} y, 0<u<b_{1},
$$

and

$$
\begin{aligned}
(\mu-\alpha) \frac{\partial V_{k, 2}\left(u ; b_{1}, b_{2}\right)}{\partial u}= & (k \delta+\lambda) V_{k, 2}\left(u ; b_{1}, b_{2}\right)+\alpha k V_{k-1,2}\left(u ; b_{1}, b_{2}\right)-\lambda\left[\int_{0}^{u-b_{1}} V_{k, 2}\left(u-y ; b_{1}, b_{2}\right) p(y) \mathrm{d} y\right. \\
& \left.+\int_{u-b_{1}}^{u} V_{k, 1}\left(u-y ; b_{1}, b_{2}\right) p(y) \mathrm{d} y\right], \quad b_{1}<u<b_{2},
\end{aligned}
$$

with boundary conditions

$$
\begin{gathered}
V_{k, 1}\left(b_{1}-; b_{1}, b_{2}\right)=V_{k, 2}\left(b_{1}+; b_{1}, b_{2}\right), \\
\left.\frac{\partial V_{k, 2}\left(u ; b_{1}, b_{2}\right)}{\partial u}\right|_{u=b_{2}-}=k V_{k-1,2}\left(b_{2} ; b_{1}, b_{2}\right), \\
\left.\mu \frac{\partial V_{k, 1}\left(u ; b_{1}, b_{2}\right)}{\partial u}\right|_{u=b_{1}-}=\left.(\mu-\alpha) \frac{\partial V_{k, 2}\left(u ; b_{1}, b_{2}\right)}{\partial u}\right|_{u=b_{1}+}+\alpha k V_{k-1,2}\left(b_{1} ; b_{1}, b_{2}\right) .
\end{gathered}
$$

Remark 4.2 Letting $k=1$, we have $V_{1}\left(u ; b_{1}, b_{2}\right)=V\left(u ; b_{1}, b_{2}\right)$, Theorem 3.3 can be reduced by Theorem 4.2. From (4.15).

$$
\left.\frac{\partial V_{1,2}\left(u ; b_{1}, b_{2}\right)}{\partial u}\right|_{u=b_{2}-}=1
$$

is an obvious result since $V_{01}\left(b_{2} ; b_{1}, b_{2}\right)=1$.

\section{The Gerber-Shiu Functions}

In the following we will discuss the famous Gerber-Shiu expected discounted penalty function $\Phi\left(u ; b_{1}, b_{2}\right)$. We also write

$$
\Phi\left(u ; b_{1}, b_{2}\right)= \begin{cases}\Phi_{1}\left(u ; b_{1}, b_{2}\right), & 0 \leq u<b_{1}, \\ \Phi_{2}\left(u ; b_{1}, b_{2}\right), & b_{1} \leq u \leq b_{2} .\end{cases}
$$

By a similar derivation to Theorem 4.1, we get the integro-differential equations and boundary conditions for $\Phi\left(u ; b_{1}, b_{2}\right)$.

Theorem 5.1 Assume that $\Phi\left(u ; b_{1}, b_{2}\right)$ is continuously differentiable in $u$ on $\left(0, b_{1}\right) \cup\left(b_{1}, b_{2}\right)$. Then, $\Phi\left(u ; b_{1}, b_{2}\right)$ satisfies the following integro-differential equations, when $0<u<b_{1}$,

$$
\mu \frac{\partial \Phi_{1}\left(u ; b_{1}, b_{2}\right)}{\partial u}=(\lambda+\delta) \Phi_{1}\left(u ; b_{1}, b_{2}\right)-\lambda\left[\int_{0}^{u} \Phi_{1}\left(u-y ; b_{1}, b_{2}\right) p(y) \mathrm{d} y+A(u)\right],
$$

and, when $b_{1}<u<b_{2}$,

$$
\begin{aligned}
(\mu-\alpha) \frac{\partial \Phi_{2}\left(u ; b_{1}, b_{2}\right)}{\partial u}= & (\lambda+\delta) \Phi_{2}\left(u ; b_{1}, b_{2}\right)-\lambda\left[\int_{0}^{u-b_{1}} \Phi_{2}\left(u-y ; b_{1}, b_{2}\right) p(y) \mathrm{d} y\right. \\
& \left.+\int_{u-b_{1}}^{u} \Phi_{1}\left(u-y ; b_{1}, b_{2}\right) p(y) \mathrm{d} y+A(u)\right],
\end{aligned}
$$

where $A(u)=\int_{u}^{\infty} \omega(u, y-u) p(y) \mathrm{d} y$ and with boundary conditions

$$
\begin{gathered}
\Phi_{1}\left(b_{1}-; b_{1}, b_{2}\right)=\Phi_{2}\left(b_{1}+; b_{1}, b_{2}\right), \\
\left.\frac{\partial \Phi_{2}\left(u ; b_{1}, b_{2}\right)}{\partial u}\right|_{u=b_{2}}=0,
\end{gathered}
$$




$$
\left.\mu \frac{\partial \Phi_{1}\left(u ; b_{1}, b_{2}\right)}{\partial u}\right|_{u=b_{1}-}=\left.(\mu-\alpha) \frac{\partial \Phi_{2}\left(u ; b_{1}, b_{2}\right)}{\partial u}\right|_{u=b_{1}+} .
$$

Proof. We can mimic the derivation of (4.1), (4.2), (4.3) and (4.5) to obtain (5.1), (5.2), (5.3) and (5.5).

Next we prove the condition (5.4). In view of the strong Markov property of the surplus process $\left\{\tilde{U}_{t}, t \geq 0\right\}$, we have

$$
\Phi\left(u ; b_{1}, b_{2}\right)=\mathrm{e}^{-\delta t} E_{u}\left[\Phi\left(\tilde{U}_{t} ; b_{1}, b_{2}\right)\right] .
$$

When the initial surplus is $b_{2}$,

$$
\begin{aligned}
\Phi_{2}\left(b_{2} ; b_{1}, b_{2}\right)= & (1-\lambda t) \mathrm{e}^{-\delta t} \Phi_{2}\left(b_{2} ; b_{1}, b_{2}\right)+\mathrm{e}^{-\delta t} \lambda t\left[\int_{0}^{b_{2}-b_{1}} \Phi_{2}\left(b_{2}-y ; b_{1}, b_{2}\right) p(y) \mathrm{d} y\right. \\
& \left.+\int_{b_{2}-b_{1}}^{b_{2}} \Phi_{1}\left(b_{2}-y, z \mathrm{e}^{-\delta t} ; b_{1}, b_{2}\right) p(y) \mathrm{d} y+\int_{b_{2}}^{\infty} \omega\left(b_{2}, y-b_{2}\right) p(y) \mathrm{d} y\right],
\end{aligned}
$$

dividing $t$ on both sides of the above expression, letting $t \rightarrow 0$, we can obtain

$$
\begin{aligned}
(\delta+\lambda) \Phi_{2}\left(b_{2} ; b_{1}, b_{2}\right)= & \lambda\left[\int_{0}^{b_{2}-b_{1}} \Phi_{2}\left(b_{2}-y ; b_{1}, b_{2}\right) p(y) \mathrm{d} y+\int_{b_{2}-b_{1}}^{b_{2}} \Phi_{1}\left(b_{2}-y, z ; b_{1}, b_{2}\right) p(y) \mathrm{d} y\right. \\
& \left.+\int_{b_{2}}^{\infty} \omega\left(b_{2}, y-b_{2}\right) p(y) \mathrm{d} y\right] .
\end{aligned}
$$

Letting $u \uparrow b_{2}$ in (5.2) and comparing it to (5.7), we obtain

$$
\left.\frac{\partial \Phi_{2}\left(u ; b_{1}, b_{2}\right)}{\partial u}\right|_{u=b_{2}-}=0 .
$$

When $u>b_{2}$, we have

$$
\Phi\left(u ; b_{1}, b_{2}\right)=\Phi_{2}\left(b_{2} ; b_{1}, b_{2}\right)
$$

thus,

$$
\left.\frac{\partial \Phi\left(u ; b_{1}, b_{2}\right)}{\partial u}\right|_{u=b_{2}+}=0
$$

So we get (5.4).

This completes the proof of Theorem 5.1.

Remark 5.1 1) In the case of $b_{1}=b_{2}$, (5.1) is corresponding to (2.6) of [3] by letting $\mu, A(u)$ substitute $c, \zeta(u)$.

2) Letting $b_{2} \rightarrow \infty$, (5.1) and (5.2) are corresponding to (3.1) of [9] by letting $\mu, A(u)$ substitute $c_{1}, \zeta(u)$.

\section{Explicit Expressions of the Laplace Transform of Ruin Time}

In this section, we give the closed form expression for the Laplace transform of ruin time when claim size has exponential distribution with mean $1 / \beta$, i.e. $p(y)=\beta \mathrm{e}^{-\beta y}, y>0$. We also write

$$
L\left(u ; b_{1}, b_{2}\right)= \begin{cases}L_{1}\left(u ; b_{1}, b_{2}\right), & 0 \leq u<b_{1}, \\ L_{2}\left(u ; b_{1}, b_{2}\right), & b_{1} \leq u \leq b_{2} .\end{cases}
$$

By setting $\omega(x, y) \equiv 1$ in (5.1) and (5.2) and letting $L\left(u ; b_{1}, b_{2}\right)$ substitute $\Phi\left(u ; b_{1}, b_{2}\right)$, we obtain the integro-differential equations and the boundary conditions satisfied by $L\left(u ; b_{1}, b_{2}\right)$ from Theorem 5.1.

Theorem 6.1 $L\left(u ; b_{1}, b_{2}\right)$ satisfies the following integro-differential equations, when $0<u<b_{1}$,

$$
\mu \frac{\partial L_{1}\left(u ; b_{1}, b_{2}\right)}{\partial u}=(\lambda+\delta) L_{1}\left(u ; b_{1}, b_{2}\right)-\lambda\left[\int_{0}^{u} L_{1}\left(u-y ; b_{1}, b_{2}\right) p(y) \mathrm{d} y+1-P(u)\right],
$$

and, when $b_{1}<u<b_{2}$, 


$$
\begin{aligned}
(\mu-\alpha) \frac{\partial L_{2}\left(u ; b_{1}, b_{2}\right)}{\partial u}= & (\lambda+\delta) L_{2}\left(u ; b_{1}, b_{2}\right)-\lambda\left[\int_{0}^{u-b_{1}} L_{2}\left(u-y ; b_{1}, b_{2}\right) p(y) \mathrm{d} y\right. \\
& \left.+\int_{u-b_{1}}^{u} L_{1}\left(u-y ; b_{1}, b_{2}\right) p(y) \mathrm{d} y+1-P(u)\right]
\end{aligned}
$$

with boundary conditions

$$
\begin{gathered}
L_{1}\left(b_{1}-; b_{1}, b_{2}\right)=L_{2}\left(b_{1}+; b_{1}, b_{2}\right), \\
\left.\frac{\partial L_{2}\left(u ; b_{1}, b_{2}\right)}{\partial u}\right|_{u=b_{2}}=0 \\
\left.\mu \frac{\partial L_{1}\left(u ; b_{1}, b_{2}\right)}{\partial u}\right|_{u=b_{1}-}=\left.(\mu-\alpha) \frac{\partial L_{2}\left(u ; b_{1}, b_{2}\right)}{\partial u}\right|_{u=b_{1}+} .
\end{gathered}
$$

Remark 6.1 In the case of $b_{2} \rightarrow \infty$, (6.1) and (6.2) are corresponding to equations (10.2) and (10.3) in [7].

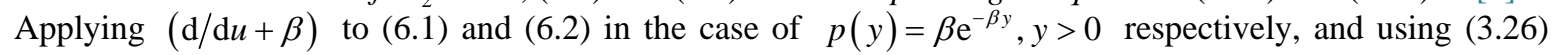
and rearranging them, we have that for $0<u<b_{1}$

$$
\mu \frac{\partial L_{1}^{2}\left(u ; b_{1}, b_{2}\right)}{\partial u^{2}}+(\beta \mu-\lambda-\delta) \frac{\partial L_{1}\left(u ; b_{1}, b_{2}\right)}{\partial u}-\beta \delta L_{1}\left(u ; b_{1}, b_{2}\right)=0,
$$

and for $b_{1}<u<b_{2}$

$$
(\mu-\alpha) \frac{\partial L_{2}^{2}\left(u ; b_{1}, b_{2}\right)}{\partial u^{2}}+[\beta(\mu-\alpha)-\lambda-\delta] \frac{\partial L_{2}\left(u ; b_{1}, b_{2}\right)}{\partial u}-\beta \delta L_{2}\left(u ; b_{1}, b_{2}\right)=0 .
$$

We can obtain the solutions of Equation (6.6) and (6.7) as follows

$$
\begin{array}{ll}
L_{1}\left(u ; b_{1}, b_{2}\right)=C_{0} \mathrm{e}^{r u}+C_{1} \mathrm{e}^{s u}, & 0 \leq u \leq b_{1}, \\
L_{2}\left(u ; b_{1}, b_{2}\right)=G_{0} \mathrm{e}^{w u}+G_{1} \mathrm{e}^{v u}, & b_{1}<u \leq b_{2},
\end{array}
$$

with the coefficients $C_{0}, C_{1}, G_{0}$ and $G_{1}$ being independent of $u$, and $r, s, w$ and $v$ are the same as in Example 3.1. Substituting (5.8) in Equation (5.1) and equating the coefficient of $\mathrm{e}^{-\beta u}$ with 0 , we have

$$
\beta\left(\frac{C_{0}}{r+\beta}+\frac{C_{1}}{s+\beta}\right)=1 .
$$

Substitute (5.8) and (5.9) in Equation (5.2) and equating the coefficient of $\mathrm{e}^{-\beta u}$ with 0 , we have

$$
C_{0} \frac{\mathrm{e}^{r b_{1}}}{r+\beta}+C_{1} \frac{\mathrm{e}^{s b_{1}}}{s+\beta}=G_{0} \frac{\mathrm{e}^{w b_{1}}}{\beta+w}+G_{1} \frac{\mathrm{e}^{v b_{1}}}{\beta+v} .
$$

From (5.3) and (5.4), we have the conditions

$$
C_{0} \mathrm{e}^{r b_{1}}+C_{1} \mathrm{e}^{s b_{1}}=G_{0} \mathrm{e}^{w b_{1}}+G_{1} \mathrm{e}^{v b_{1}},
$$

and

$$
G_{0} w \mathrm{e}^{w b_{2}}+G_{1} v \mathrm{e}^{v b_{2}}=0 .
$$

It follows from (6.11) and (6.12) that

$$
\begin{aligned}
& C_{0}=\frac{\beta+r}{r-s}\left(\mathrm{e}^{w b_{1}} \frac{w-s}{\beta+w} G_{0}+\mathrm{e}^{v b_{1}} \frac{v-s}{\beta+v} G_{1}\right) \mathrm{e}^{-r b_{1}}, \\
& C_{1}=\frac{\beta+r}{s-r}\left(\mathrm{e}^{w b_{1}} \frac{w-r}{\beta+w} G_{0}+\mathrm{e}^{v b_{1}} \frac{v-r}{\beta+v} G_{1}\right) \mathrm{e}^{-s b_{1}},
\end{aligned}
$$

and from (6.13), we get 


$$
G_{0}=-(v / w) \mathrm{e}^{(v-w) b_{2}} G_{1} .
$$

Substituting (6.14), (6.15) into (6.10) and then using (6.16), the constants $G_{0}$ and $G_{1}$ can be given by

$$
\begin{gathered}
G_{0}=\frac{r-s}{\beta} \frac{v(\beta+w)(\beta+v)}{I} \mathrm{e}^{b_{2}(v-w),} \\
G_{1}=\frac{s-r}{\beta} \frac{w(\beta+w)(\beta+v)}{I},
\end{gathered}
$$

where

$$
\begin{aligned}
I= & (\beta+w) w\left[(v-r) \mathrm{e}^{-s b_{1}}-(v-s) \mathrm{e}^{-r b_{1}}\right] \mathrm{e}^{v b_{1}} \\
& +(\beta+v) v\left[(w-s) \mathrm{e}^{-r b_{1}}-(w-r) \mathrm{e}^{-s b_{1}}\right] \mathrm{e}^{w b_{1}+(v-w) b_{2}} .
\end{aligned}
$$

Substituting (6.17) and (6.18) into (6.14) and (6.15), the constants $C_{0}$ and $C_{1}$ can be given by

$$
\begin{aligned}
& C_{0}=\frac{\beta+r}{\beta I}\left[v(\beta+v)(w-s) \mathrm{e}^{w b_{1}+(v-w) b_{2}}-w(\beta+w)(v-s) \mathrm{e}^{v b_{1}}\right] \mathrm{e}^{-r b_{1}}, \\
& C_{1}=\frac{\beta+s}{\beta I}\left[-v(\beta+v)(w-r) \mathrm{e}^{w b_{1}+(v-w) b_{2}}+w(\beta+w)(v-r) \mathrm{e}^{v b_{1}}\right] \mathrm{e}^{-s b_{1}} .
\end{aligned}
$$

From (6.17)-(6.21), we have

$$
\begin{aligned}
L_{1}\left(u ; b_{1}, a\right)= & \frac{\beta+r}{\beta I}\left[v(\beta+v)(w-s) \mathrm{e}^{w b_{1}+(v-w) b_{2}}-w(\beta+w)(v-s) \mathrm{e}^{v b_{1}}\right] \mathrm{e}^{r\left(u-b_{1}\right)} \\
& +\frac{\beta+s}{\beta I}\left[-v(\beta+v)(w-r) \mathrm{e}^{w b_{1}+(v-w) b_{2}}+w(\beta+w)(v-r) \mathrm{e}^{v b_{1}}\right] \mathrm{e}^{s\left(u-b_{1}\right)}, \quad \text { if } 0 \leq u<b_{1}, \\
L_{2}\left(u ; b_{1}, a\right)= & \frac{s-r}{w} \beta I v(\beta+w)(\beta+v) \mathrm{e}^{w u+(v-w) b_{2}}+\frac{r-s}{\beta I}(\beta+w)(\beta+v) \mathrm{e}^{v u}, \quad \text { if } b_{1} \leq u \leq b_{2} .
\end{aligned}
$$

Remark 6.2 Letting $b_{2} \rightarrow \infty$, from (6.17) to (6.21), we have

$$
\begin{gathered}
\lim _{b_{2} \rightarrow \infty} C_{0}=\frac{\beta+r}{\beta} \frac{(w-s) \mathrm{e}^{s b_{1}}}{(w-s) \mathrm{e}^{s b_{1}}-(w-r) \mathrm{e}^{r b_{1}}}, \\
\lim _{b_{2} \rightarrow \infty} C_{1}=\frac{\beta+s}{\beta} \frac{(r-w) \mathrm{e}^{r b_{1}}}{(w-s) \mathrm{e}^{s b_{1}}-(w-r) \mathrm{e}^{r b_{1}}}, \\
\lim _{b_{2} \rightarrow \infty} G_{0}=\frac{r-s}{\beta} \frac{\beta+w}{(w-s) \mathrm{e}^{-r b_{1}}-(w-r) \mathrm{e}^{-s b_{1}}} \mathrm{e}^{-w b_{1}}, \\
\lim _{b_{2} \rightarrow \infty} G_{1}=0 .
\end{gathered}
$$

Thus,

$$
\begin{gathered}
\lim _{b_{2} \rightarrow \infty} L_{1}\left(u ; b_{1}, b_{2}\right)=\frac{1}{\beta} \frac{(\beta+r)(w-s) \mathrm{e}^{s b_{1}+r u}+(\beta+s)(r-w) \mathrm{e}^{r b_{1}+s u}}{(w-s) \mathrm{e}^{s b_{1}}-(w-r) \mathrm{e}^{r b_{1}}}, \quad 0 \leq u \leq b_{1}, \\
\lim _{b_{2} \rightarrow \infty} L_{2}\left(u ; b_{1}, b_{2}\right)=\frac{r-s}{\beta} \frac{\beta+w}{(w-s) \mathrm{e}^{-r b_{1}}-(w-r) \mathrm{e}^{-s b_{1}}} \mathrm{e}^{w\left(u-b_{1}\right)}, \quad u>b_{1},
\end{gathered}
$$

which are (10.17) and (10.19) of [7].

\section{Acknowledgements}

The authors are grateful to the anonymous referee's careful reading and detailed helpful comments and con- 
structive suggestions, which have led to a significant improvement of the paper. The research was supported by the National Natural Science Foundation of China (No. 11171179), the Research Fund for the Doctoral Program of Higher Education of China (No. 20133705110002) and the Program for Scientific Research Innovation Team in Colleges and Universities of Shandong Province.

\section{References}

[1] Finetti, B.D. (1957) Su un impostazione alternativa della teoria collectiva del rischio. Transactions of the 15th International Congress of Applied Probability, 41, 117-130.

[2] Gerber, H.U. and Shiu, E.S.W. (1998) On the Time Value of Ruin. North American Actuarial Journal, 2, 48-78. http://dx.doi.org/10.1080/10920277.1998.10595671

[3] Lin, X.S., Willmot, G.E. and Drekic, S. (2003) The Classical Risk Model with a Constant Dividend Barrier: Analysis of the Gerber-Shiu Discounted Penalty Function. Insurance: Mathematics and Economics, 33, 551-566. http://dx.doi.org/10.1016/j.insmatheco.2003.08.004

[4] Gerber, H.U. and Shiu, E.S.W. (2004) Optimal Dividends: Analysis with Brownian Motion. North American Actuarial Journal, 8, 1-20. http://dx.doi.org/10.1080/10920277.2004.10596125

[5] Jeanblang-Picque, M. and Shiryaev, A.N. (1995) Optimization of the Flow of Dividends. Russian Mathematical Surveys, 20, 257-277. http://dx.doi.org/10.1070/RM1995v050n02ABEH002054

[6] Asmussen, S. and Taksar, M. (1997) Controlled Diffusion Models for Optimal Dividend Pay-Out. Insurance: Mathematics and Economics, 20, 1-15. http://dx.doi.org/10.1016/S0167-6687(96)00017-0

[7] Gerber, H.U. and Shiu, E.S.W. (2006) On Optimal Dividend Strategy in the Compound Poisson Model. North American Actuarial Journal, 10, 76-93. http://dx.doi.org/10.1080/10920277.2006.10596249

[8] Gerber, H.U. and Shiu, E.S.W. (2006) On Optimal Dividends: From Reflection to Refraction. Journal of Computational and Applied Mathematics, 186, 4-22. http://dx.doi.org/10.1016/j.cam.2005.03.062

[9] Lin, X.S. and Pavlova, K.P. (2006) The Compound Poisson Risk Model with a Threshold Dividend Strategy. Insurance: Mathematics and Economics, 38, 57-80. http://dx.doi.org/10.1016/j.insmatheco.2005.08.001

[10] Wan, N. (2007) Dividend Payments with a Threshold Strategy in the Compound Poisson Risk Model Perturbed by Diffusion. Insurance: Mathematics and Economics, 40, 509-523. http://dx.doi.org/10.1016/j.insmatheco.2006.08.002

[11] Ng, A.C.Y. (2009) On a Dual Model with a Dividend Threshold. Insurance: Mathematics and Economics, 44, 315-324. http://dx.doi.org/10.1016/j.insmatheco.2008.11.011

[12] Fang, Y. and Wu, R. (2008) Optimal Dividends in the Brownian Motion Risk Model with Interest. Journal of Computational and Applied Mathematics, 229, 145-151. http://dx.doi.org/10.1016/j.cam.2008.10.021

[13] Chi, Y.C. and Lin, X.S. (2011) On the Threshold Dividend Strategy for a Generalized Jump-Diffusion Risk Model. Insurance: Mathematics and Economics, 48, 326-337. http://dx.doi.org/10.1016/j.insmatheco.2010.11.006

[14] Yin, C.C. and Wen, Y.Z. (2013) An Extension of Paulsen-Gjessing’s Risk Model with Stochastic Return on Investments. Insurance: Mathematics and Economics, 52, 469-476. http://dx.doi.org/10.1016/j.insmatheco.2013.02.014

[15] Albrecher, H. and Hartinger, J. (2007) A Risk Model with Multi-Layer Dividend Strategy. North American Actuarial Journal, 11, 43-64. http://dx.doi.org/10.1080/10920277.2007.10597447

[16] Lin, X.S. and Sendova, K.P. (2008) The Compound Poisson Risk Model with Multiple Threshold. Insurance: Mathematics and Economics, 42, 617-627. http://dx.doi.org/10.1016/j.insmatheco.2007.06.008

[17] Jiang, W.Y., Yang, Z.J. and Li, X.P. (2012) The Discounted Penalty Function with Multi-Layer Dividend Strategy in the Phase-Type Risk Model. Insurance: Mathematics and Economics, 82, 1358-1366.

[18] Ng, A.C.Y. (2010) On the Upcrossing and Downcrossing Probabilities of a Dual Risk Model with Phase-Type Gains. Astin Bulletin, 40, 281-306. http://dx.doi.org/10.2143/AST.40.1.2049230

[19] Wang, C.W., Yin, C.C. and Li, E.Q. (2010) On the Classical Risk Model with Credit and Debit Interests under Absolute Ruin. Statistics and Probability Letter, 80, 427-436. http://dx.doi.org/10.1016/j.spl.2009.11.020

[20] Liu, D.H. and Liu, Z.M. (2011) The Perturbed Compound Poisson Risk Model with Linear Dividend Barrier. Journal of Computational and Applied Mathematics, 235, 2357-2363. http://dx.doi.org/10.1016/j.cam.2010.10.034

[21] Gao, S. and Liu, Z.M. (2010) The Perturbed Compound Poisson Risk Model with Constant Interest and a Threshold Dividend Strategy. Journal of Computational and Applied Mathematics, 233, 2181-2188. http://dx.doi.org/10.1016/j.cam.2009.10.004 
Scientific Research Publishing (SCIRP) is one of the largest Open Access journal publishers. It is currently publishing more than 200 open access, online, peer-reviewed journals covering a wide range of academic disciplines. SCIRP serves the worldwide academic communities and contributes to the progress and application of science with its publication.

Other selected journals from SCIRP are listed as below. Submit your manuscript to us via either submit@scirp.org or Online Submission Portal.
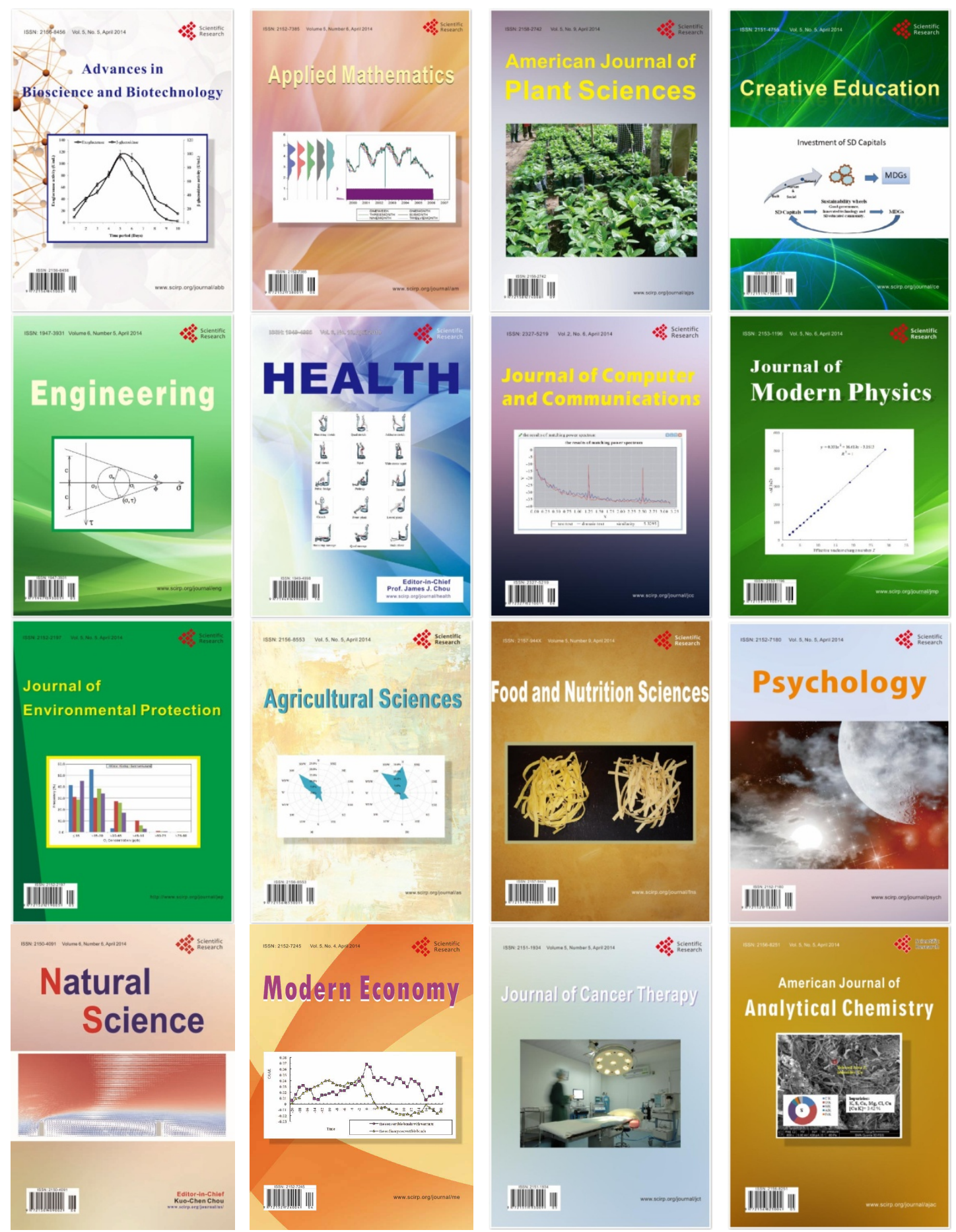